Results 3 polymorphisms (A46G, C79G and A523C) and a mutation (C491T) of ADRB2 were found. Genotype distributions of A523C in control and hypertriglyceridemia, control and hypo-HDL-c individuals were different $(p<0.05)$. Logistic regression analysis showed that the OR for hypertension was 2.894 in C523C group against the $A 523 A+A 523 C$ group $(p=0.009)$; compared the A523A+A523C group, the OR for hypertriglyceridemia in C523C group were 2.666 $(p=0.031)$. The frequency of C491T was $1.2 \%(n=5)$ and no TT genotype was found. That C491T was found only in hypertensive or dyslipidemia group. All five subjects were diagnosis with hypertension and dyslipidemia. Four out of five individuals with C491T had Hyper-LDL-C. The mean blood pressure and serum LDL-C of the five subjects were much higher than normal value.

Conclusions Variation of ADRB2 may play a causal role in the pathogenesis of the hypertension and dyslipidemia in Xinjiang Kazakans.

\section{e0167 THE RELATIONSHIP STUDY BETWEEN T663A POLYMORPHISM OF QENAC GENE WITH ESSENTIAL HYPERTENSION AND SERUM ELECTROLYTES IN XINJIANG KAZAKHS}

doi:10.1136/hrt.2010.208967.167

Li Nanfang, Xu Hong, Yao Xiaoguang, Yan Zhitao. Hypertension Unit of The People's Hospital of Xinjiang Uygur Autonomous Region, The Institute of Hypertension of Xinjiang, The Center of Hypertension of The People's Hospital of Xinjiang Uygur Autonomous Region, Urumqi, China

Introduction To study the relationship between the T663A polymorphism in $\alpha \mathrm{ENaC}$ gene with essential hypertension $(\mathrm{EH})$ and serum electrolytes of Xinjiang Kazakhs.

Methods 516 Xinjiang Kazakhs aged elder than 30 years were recruited in this population based on case-control study from the patural area of Xinjiang, and were divided into hypertensives $(\mathrm{EH}$ group) and normotensives (NT group). The gene polymorphism of T663A by PCR-RFLP and the serum electrolytes were examined.

Results The frequencies of AA, AG, GG genotypes were $15.7 \%$, $50.2 \%, 34.1 \%$ and the frequencies of alleles A, G were $40.8 \%, 59.2 \%$, respectively. The frequencies of AA, AG, GG were $16.3 \%, 49.1 \%$, $34.6 \%$ in $\mathrm{EH}$ group and $15.1 \%, 51.4 \%, 33.5 \%$ in NT group, respectively. The distribution of genotypic and allelic frequencies were not different between $\mathrm{EH}$ group and NT group $(\mathrm{p}=0.85 ; \mathrm{p}=1.0)$. No significant difference in levels of blood pressure and $\mathrm{K}^{+}, \mathrm{Na}^{+} / \mathrm{K}^{+}$ between subjects among genotypes. The T663A polymorphism were excluded as independent variables controlling age and BMI. But the serum $\mathrm{Na}^{+}$of the subjects with AA genotype was higher than those subjects with AG and GG genotypes $(p=0.032)$.

Conclusions T663A polymorphism might not be associated with $\mathrm{EH}$ but the AA genotype of T663A polymorphisms might be associated with the higher level of the serum $\mathrm{Na}^{+}$of Xinjiang Kazakhs.

\section{e0168 THE RELATIONSHIP STUDY BETWEEN T3593C POLYMORPHISM OF $\alpha$ ENAC GENE WITH ESSENTIAL HYPERTENSION AND SERUM ELECTROLYTES IN XINJIANG KAZAKHS}

doi:10.1136/hrt.2010.208967.168

Li Nanfang, Xu Hong, Yan Zhitao, Wang Hongmei. Hypertension Unit of The People's Hospital of Xinjiang Uygur Autonomous Region, The Institute of Hypertension of Xinjiang, The Center of Hypertension of The People's Hospital of Xinjiang Uygur Autonomous Region, Urumqi, China

Introduction To study the relationship between T3593C polymorphism in the $\alpha$ subunit gene of epithelial sodium channel
$(\mathrm{ENaC})$ with essential hypertension $(\mathrm{EH})$ and serum electrolytes of Xinjiang Kazakhs.

Methods More than 500 Xinjiang Kazakhs aged more than 30 years were recruited by cluster sampling from the pasture area of Xinjiang and were divided into hypertensives (EH group) and normotensives (NT group). The genotypes of T3593C were determined by PCRRFLP method and the serum electrolytes were measured.

Results The frequencies of TT, TC, CC genotypes were $88.39 \%$, $10.63 \%, 0.98 \%$ and the alleles frequencies of $\mathrm{T}$, C were $93.7 \%, 6.3 \%$, respectively. The distributed genotypic frequencies of TT, TC, CC were $89.33 \%, 9.88 \%, 0.79 \%$ in $\mathrm{EH}$ group and $87.45 \%, 11.37 \%, 1.18 \%$ in NT group, respectively. The genotypic and allelic frequencies were not different between $\mathrm{EH}$ group and NT group ( $p=0.78 ; p=0.46)$. There was no significant difference in levels of blood pressure and serum electrolytes between subjects with the TT and TC+CC genotypes ( $p>0.05$ ). The T3593C polymorphism were excluded as independent variables which related to the blood pressure and serum electrolytes of subjects controlling FPR age and gender by multiple logistic analysis. Conclusions The T3593C polymorphism might not be associated with EH and serum electrolytes of Xinjiang Kazakhs.

\section{e0169 IN VITRO EFFECTS OF LOW MOLECULAR WEIGHT HEPARIN ON CLOT RATE}

doi:10.1136/hrt.2010.208967.169

Shi Xu-bo, Zheng Hua, Biao Hong, Li Chao, Yang Yi. Department of Cardiology, Beijing Tong Ren Hospital, Beijing, China

Objective This study was designed to determine the in vitro effects of different doses and different kinds of Low Molecular Weight Heparin (LMWH) on clot rate (CR), and to determine whether the CR can be used to monitor LMWH.

Methods This study was performed in two phases in vitro. The first, $\mathrm{CR}$ was measured with different reagent (glass beads, celite and kaolin) on volunteer $(\mathrm{n}=20)$ blood samples spiked with increasing concentrations of LMWH (dalteparin, $0.2-1.8 \mathrm{IU} / \mathrm{ml}$ ). The second, $\mathrm{CR}$ were measured with the same reagent (glass beads) on volunteer $(n=20)$ blood samples spiked with the same concentrations (0.8 IU/ $\mathrm{ml}$ ) of different LMWH (dalteparin, enoxaparin and nadroparin). Regression analysis was performed to establish a regression equation from corresponding anti-Xa LMWH levels.

Results With the increase in doses of dalteparin, CR values were reduced gradually and an exponential relationship was observed between the CR values and dalteparin concentrations $(p<0.01)$ for all three reagents. With the same concentrations $(0.8 \mathrm{IU} / \mathrm{ml})$ of LMWH, dalteparin showed a more effect on CR (dalteparin $7.4 \mathrm{IU} /$ min vs enoxaparin $8.5 \mathrm{IU} / \mathrm{min}$, nadroparin $8.5 \mathrm{IU} / \mathrm{min}, \mathrm{p}<0.05$ ). Compare with the baseline $(17.6 \mathrm{IU} / \mathrm{min})$, all three kinds of $\mathrm{LMWH}$ induced a significant change in the CR $(p<0.01)$.

Conclusions The in vitro studies have shown that, there was an exponential relationship between the CR and dalteparin concentrations for all three reagents. All three kinds of LMWH could significantly reduce the value of $\mathrm{CR}$. CR test may be a suitable method for monitoring the anticoagulant effect of LMWH.

\section{e0170 THE SENSITIVITY OF DIFFERENT REAGENTS FOR LABORATORY MONITORING OF LOW MOLECULAR WEIGHT HEPARIN: AN IN VITRO STUDY}

doi:10.1136/hrt.2010.208967.170

Shi Xu-Bo, Hu Da-Yi, Wang Jian-Qi, Chen Zhe, Yu Hai-Rong, Bai Ying. Department of Cardiology, Beijing Tong Ren Hospital, Beijing, China

Objective Because of the lack of Point-of-care testing, the use of low-molecular-weight heparin (LMWH)therapy in some special 